\title{
Clomipramine Could Be Useful in Preventing Neurological Complications of SARS-CoV-2 Infection
}

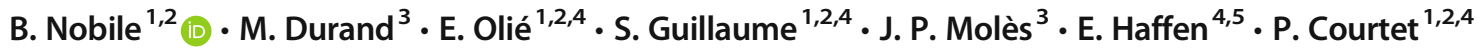

Received: 2 June 2020 / Accepted: 23 June 2020 / Published online: 29 June 2020

(C) Springer Science+Business Media, LLC, part of Springer Nature 2020

Dear Dr. Gendelman,

The global pandemic of the new coronavirus disease 2019 (COVID-19) has affected more than 7 million of people and provoked more than 400,000 deaths worldwide. In view of the urgent need of efficient treatments and the time to develop specific antiviral drugs, drug repositioning seems to be a good compromise (Sanders et al. 2020). Among the different aspects of COVID-19 physiopathology, the excessive inflammation following infection rapidly emerged as an important issue. Indeed, the significant damages in lungs and other organs observed are mainly explained by amplified immune response and cytokine release (Sanders et al. 2020). Tocilizumab (a humanized anti-IL-6 monoclonal antibody) has shown promising effects in reducing COVID-19 severity and mortality (Sanders et al. 2020). This suggests that targeting the "cytokine storm" seems to be efficient.

On the other hand, SARS-CoV-2 was found in the cerebrospinal fluid of infected patients; additionally partial neuronal degeneration was observed in deceased patients (Wu et al. 2020). This tropism for the central nervous system (CNS) is also reflected by some COVID-19 symptoms, such as anosmia, dysgeusia, agitation, confusion, epilepsy, ischemic

B. Nobile

benedicte.nobile@gmail.com

1 Department of Emergency Psychiatry and Acute Care, CHU Montpellier, Montpellier, France

2 PSNREC, INSERM, CHU de Montpellier, University Montpellier, Montpellier, France

3 Pathogenesis and control of chronic infection, INSERM, EFS; CHU Montpellier, Montpellier, University of Montpellier, Montpellier, France

4 FondaMental Foundation, Créteil, France

5 Service de Psychiatrie de l'Adulte, CIC-1431 INSERM, CHU de Besançon, Laboratoire de Neurosciences, Université de Franche-Comté, UBFC, Besançon, France attacks, cognitive impairment (sometimes severe), and also encephalitis, leading to brain inflammation and lesions $(\mathrm{Wu}$ et al. 2020). In addition, brain damage caused by viral infection could lead to psychiatric consequences. Indeed, other coronaviruses have already been associated with the emergence of psychiatric disorders (e.g. psychosis, major depression) (Severance et al. 2011). This could be the consequence of the high inflammation induced by the infection. Effectively, an increase in cytokine levels reduces serotonin bioavailability, inhibits dopamine synthesis, increases glutamate release from astrocytes, alters the negative feedback of the hypothalamic-pituitary-adrenal axis and also impact neuroplasticity (Bauer and Teixeira 2019). Deregulation of these systems and inflammation have been associated with psychiatric disorders such as depression, bipolar disorder, schizophrenia and suicidal behavior (Bauer et Teixeira 2019). Moreover, it has been hypothesized that SARS-CoV-2 affinity for ACE-2 receptors could lead to a decrease in serotonin and dopamine bioavailability (as previously reported for SARS-CoV), thus contributing to psychiatric risk. Consequently, it is crucial to find molecules that pass easily through the blood brain barrier (BBB) and diffuse largely in the CNS at therapeutic concentrations to prevent brain inflammation and reduce neurological complications of SARS-CoV-2 infection.

The tricyclic antidepressant clomipramine, a serotonin and noradrenaline reuptake inhibitor, is one of the only antidepressant with constant anti-inflammatory properties at therapeutic concentration as shown in many studies (in vitro, in vivo and humans studies) (Baumeister et al. 2016). At a peripheral level, clomipramine provokes a decrease of pro-inflammatory cytokines levels such as Interleukin-6 (IL-6), IL-1 $\beta$ and Tumor Necrosis Factor $\alpha(\mathrm{TNF} \alpha)$ and an increase of the anti-inflammatory cytokine IL-10 (Baumeister et al. 2016). In addition studies demonstrated that clomipramine has antiinflammatory effect also in the CNS. Indeed, clomipramine was found to significantly reduces lipopolysaccharideinduced acute inflammation and displays a neuroprotective effect by attenuating microglia toxicity and by acting on 
macrophages and astrocytes in microglial cells co-cultured with neurons (Hwang et al. 2008). This is all the most interesting since microglia and astrocytes are the main mediators of neuroinflammation. In this study, administration of clomipramine at therapeutic concentrations also decreased the production of TNF $\alpha$ and nitric oxide, and the mRNA expression of nitric oxide synthase, IL-1 $\beta$ and TNF $\alpha$. Moreover, clomipramine inhibited the activation of the NF-KB and p38 MAPK pro-inflammatory pathways. Another study combining in vitro and in vivo experiences demonstrated that clomipramine exerts its anti-inflammatory action in the brain by also inhibiting the nucleotide-binding oligomerization domain leucine-rich repeat-containing family pyrin domaincontaining 3 (NLRP3) inflammasome. Its inhibition by clomipramine led to a significant decrease of TNF $\alpha$, IL-1 $\beta$, IL-6 as well as IL-1 $\beta$ and IL-6 gene expression (Gong et al. 2019). Furthermore, clomipramine has been proposed as a candidate for the treatment of progressive multiple sclerosis, a severe brain inflammatory disease, due to its strong antiinflammatory properties in the CNS at therapeutic concentrations (Faissner et al. 2017). Finally, clomipramine interacts with nicotinic receptors, involved in anti-inflammatory process and potentially implicated in SARS-CoV-2 physiopathology. Although the interaction between SARS-CoV-2 and nicotinic receptors is not elucidated, we could hypothesize that clomipramine contributes to inflammation modulation also by inhibiting the interaction between SARS-CoV-2 and nicotinic receptors, additional studies are needed.

In view of those data, we hypothesized that clomipramine could be a good candidate since this molecule has strong antiinflammatory properties, passes easily the BBB and accumulates in the CNS (12.5-fold higher concentration than in plasma or serum) (Hwang et al. 2008). Thus, clomipramine could prevent the brain damage caused by SARS-CoV-2. Other antidepressants have anti-inflammatory properties, but we focused on clomipramine for different reasons. First, clomipramine was one of the only antidepressant with constant antiinflammatory properties in all studies. Second, clomipramine inhibits brain inflammation and has been proposed as a potential treatment for progressive multiple sclerosis. Finally, in some studies screening large panels of molecules, clomipramine significantly inhibited replication of SARS-CoV and MERS-CoV (Dyall et al. 2017). The mechanisms of this effect were not determined and further studies are needed to assess its potential action on viral replication.

Clomipramine is on the list of the essential medicines of the World Health Organization, showing its importance and safety. This molecule can have side effects, including weight increase, sedation, hypotension, and anticholinergic effects. Nevertheless, its side effects are less severe and dangerous than those of some of the currently tested molecules (e.g. hydroxychloroquine). Finally, clomipramine has already been given to healthy volunteers (without psychiatric disorder), and was well tolerated without mood changes at therapeutic concentration (Cerqueira et al. 2014).

In conclusion, clomipramine could be a good candidate to counteract SARS-CoV-2 infection worsening thanks to its high penetration in the brain and its anti-inflammatory effects and need to be assessed. Some other antidepressants that are better tolerated than clomipramine could also have anti-inflammatory properties and should be evaluated. It may be possible to envisage collaborative studies between psychiatrists (who routinely prescribe these molecules), virologists, immunologists and intensive care specialists to test their effects.

\section{References}

Bauer ME, Teixeira AL (2019) Inflammation in psychiatric disorders: what comes first?: inflammation in psychiatric disorders. Ann N Y Acad Sci 1437(1):57-67

Baumeister D, Ciufolini S, Mondelli V (2016) Effects of psychotropic drugs on inflammation: consequence or mediator of therapeutic effects in psychiatric treatment? Psychopharmacology 233(9):1575-1589

Cerqueira CT, Sato JR, de Almeida JRC, Amaro E, Leite CC, Gorenstein C, Gentil V, Busatto GF (2014) Healthy individuals treated with clomipramine: an FMRI study of brain activity during autobiographical recall of emotions. Transl Psychiatry 4(7):e405-e405

Dyall J, Gross R, Kindrachuk J, Johnson RF, Olinger GG, Hensley LE, Frieman MB, Jahrling e PB (2017) Middle East respiratory syndrome and severe acute respiratory syndrome: current therapeutic options and potential targets for novel therapies. Drugs 77(18): 1935-1966

Faissner, Simon, Manoj Mishra, Deepak K. Kaushik, Jianxiong Wang, Yan Fan, Claudia Silva, Gail Rauw, Luanne Metz, Marcus Koch, et V. Wee Yong. 2017. « Systematic Screening of Generic Drugs for Progressive Multiple Sclerosis Identifies Clomipramine as a Promising Therapeutic ». Nature Communications 8(1)

Gong W, Zhang S, Zong Y, Halim M, Ren Z, Wang Y, Ma Y, Li B, Ma L, Zhou G, Yu J, Zhang J, Liu Q (2019) Involvement of the microglial NLRP3 Inflammasome in the anti-inflammatory effect of the antidepressant clomipramine. J Affect Disord 254:15-25

Hwang J, Zheng LT, Ock J, Lee MG, Kim S-H, Lee H-W, Lee W-H, Park $\mathrm{H}-\mathrm{C}$, Suk K (2008) Inhibition of glial inflammatory activation and neurotoxicity by tricyclic antidepressants. Neuropharmacology 55(5):826-834

Sanders, James M., Marguerite L. Monogue, Tomasz Z. Jodlowski, et James B. Cutrell. 2020. «Pharmacologic treatments for coronavirus disease 2019 (COVID-19): a review ». JAMA

Severance EG, Dickerson FB, Viscidi RP, Bossis I, Stallings CR, Origoni AE, Sullens A, Yolken RH (2011) Coronavirus Immunoreactivity in individuals with a recent onset of psychotic symptoms. Schizophr Bull 37(1):101-107

Wu, Yeshun, Xiaolin Xu, Zijun Chen, Jiahao Duan, Kenji Hashimoto, Ling Yang, Cunming Liu, et Chun Yang. 2020. «nervous system involvement after infection with COVID-19 and other coronaviruses ». Brain, Behavior, and Immunity

Publisher's Note Springer Nature remains neutral with regard to jurisdictional claims in published maps and institutional affiliations. 